Pacific Journal of Mathematics

SECOND NOTE ON DAVID HARRISON'S THEORY OF

D. W. DUE OS 


\title{
SECOND NOTE ON DAVID HARRISON'S THEORY OF PREPRIMES
}

\author{
D. W. Dubois
}

Some classes of partially ordered rings are studied by means of representations in $C(X)$, where $X$ is a compact Hausdorff space. The first theorem generalizes the characterizations, due, respectively, to Harrison and the author, of the subrings of the real field, and the subrings of $C(X)$. Among the many consequences proved are the following:

1. If $A$ is a simple ring (no two-sided ideals) partially ordered so that (a) if $n$ is a positive integer and $n x \geqq 0$, then $x \geqq 0$; and (b) for all $x$ in $A$ there exists an integer $n$ exceeding $x$; then $A$ is a commutative field.

2 . Let $F$ be a field, let $P$ be a conic prime in $F$ whose intersection $P_{c}$ in the center $C$ of $F$ is a primitive $(A C)$ cone in $C$. Then $P_{c}$ is an Archimedean order in $C$.

3. The compact Hausdorff space $X$ admits a base of power at most $2^{\aleph_{0}}$ of open-and-closed sets if and only if $C(X)$ contains a dense subfield.

We prove three theorems and many corollaries on infinite preprimes with Archimedean conditions. Theorem 1 generalizes the main result of our first note on preprimes, Theorem 2 relates two Archimedean conditions for infinite primes, and Theorem 3 is concerned with infinite preprimes in a field. The corollaries to Theorem 1 generalize Hilbert's Theorem that an Archimedean ordered field is commutative; these tend to confirm (but see also the examples) our conjecture that any primitive $(A C)$ conic prime in a field is an order. An application is given in the corollary to Theorem 3 which characterizes a large class of totally disconnected compact Hausdorff spaces by a property (that makes no reference to partial order or preprimes) of the rings of continuous real functions on the spaces. A rather large collection of examples is given to show that conditions in the theorems are not superfluous. For furnishing us with encouragement, ideas and unpublished notes we are very grateful to David Harrison, whose influence shows (we hope) throughout this note.

The best historical account we can give of the problems studied here is a chronological list of theorems needed later. See $\S 2 \mathrm{~b}$, or our previous note for the definitions. Although Theorem 02 is not precisely equivalent to any statement in the first three references it is easily deduced by methods of each of them; it is proved in the fourth reference by a routine Zorn's lemma argument. We denote by $X$ a compact Hausdorff space and by $C(X)$ the partially ordered topological ring (or 
real algebra) of all continuous real functions on $X$, with the topology of uniform convergence and with $f \geqq g$ if and only if $f(x) \geqq g(x)$ holds for all $x$ in $X$. The theorems are stated in our terminology; Stone added a completeness condition to the hypotheses of Theorem 03 but noted that it was not really essential to the method of proof. We denote by $N$ the set of all positive integers.

THEOREM 01. (Hilbert [9].) If a field contains an Archimedean order then it is commutative.

Theorem 02. (Artin [1]; Artin-Schreier [2]; Baer [3]; cf. [4].) Let $P$ be a conic division preprime containing all squares in the commutative field $F$. Then $P$ is the intersection of all the orders in $F$ that contain $P$.

THEOREM 03. (Stone [11]). Let $A$ be a commutative ring with unit 1 such that for all $n$ in $N$ there exists $x$ in $A$ with $n x=1$. Let $\langle A, P\rangle$ be a Stone ring, where $P$ contains all squares in $A$. Then $\langle A, P\rangle$ is order-isomorphic with a subring of $C(X)$.

THEOREM 04. (Kadison [10].) Every complete Archimedean ordered algebra is a $C(X)$.

THEOREM 05. (Dubois [4].) If $P$ is a primitive $(A C)$ division cone in a commutative field $F$ then $P$ is an intersection of orders in $F$.

THEOREM 06. (Harrison [7].) A prime is finite if and only if it is a subring.

THEOREM 07. (Harrison [7].) Let $P$ be a prime in the commutative ring $A$, and let $u$ be a unit of $A$ belonging to $P$. Then $u^{-1}$ belongs to $P$ if and only if $-u$ does not belong to $P$.

THEOREM 08. (Harrison [7]; see also [5].) If $A$ is partially ordered by a real infinite Archimedean conic prime then $A$ is orderisomorphic with a subring of the real field $R$.

THEOREM 09. (Dubois [5]; uniqueness is conjectured by Harrison [8].) Let $\langle A, P\rangle$ be a Stone ring. Then there exists a unique compact Hausdorff space $X=X_{A}$ satisfying: The ring $C(X)$ contains a subring $S$ which is order-isomorphic with $\langle A, P\rangle$ and whose ring $S_{N}$ of fractions with respect to $N$ is dense in $C(X)$. 
Proof. (of uniqueness). Let $X$ and $X^{\prime}$ be compact Hausdorff spaces, let $S$ and $S^{\prime}$ be subrings of $C(X)$ and $C\left(X^{\prime}\right)$, respectively, such that $S_{N}$ and $S_{N}^{\prime}$ are dense in $C(X)$ and $C\left(X^{\prime}\right)$ and let $\varphi$ be an orderisomorphism of $S$ onto $S^{\prime}$. The canonical map $\varphi_{N}: S_{N} \rightarrow S_{N}^{\prime}$ is also an order-isomorphism. The density conditions imply that $\varphi_{N}$ extends uniquely to a bicontinuous order-isomorphism of $C(X)$ onto $C\left(X^{\prime}\right)$. By [6; Th. 4.9] $X$ and $X^{\prime}$ are homeomorphic.

\section{New theorems and definitions.}

a. Statements of the theorems corollaries. In these theorems we are using 'Archimedean' in a different sense from the previous note. We say that $P$ is Archimedean, where $P$ is an infinite preprime, when for every $p$ in $P$, there exists an integer $n$ with $n-p$ in $P$. The definitions are given after the statements of the theorems.

THEOREM 1. Let $P$ be an infinite $N$-cancellation preprime in the ring $A$, let $B=B_{P}, J=J_{P}$.

A. c.f. Harrison [8]. If $P$ is Archimedean then $P^{*}$ is an infinite Archimedean $N$-cancellation (AC) preprime. If $P$ is full Archimedean then $P^{*}$ is also full Archimedean.

B. If $P$ is full Archimedean then $\left\langle A / J, P^{*} / J\right\rangle$ is a Stone ring. Hence $A / J$ is commutative.

C. Let $P_{1}=P \cap B$, considered as a preprime in $B$. Then $\left\langle B / J, P_{1}^{*} / J\right\rangle$ is a Stone ring.

D. If $P$ is Archimedean then $\left\langle(P-P) / J, P^{*} / J\right\rangle$ is a Stone ring.

Corollary 1. Let $P$ be an $N$-cancellation infinite preprime in the ring $A$. Each of the following conditions implies that $A$ is commutative:

A. For every $x$ in $A, 1+x^{2}$ is invertible, and $P$ is an (AW) cone containing all squares in $A$. in $A$.

B. $A$ is a field and $P$ is an (AW) cone containing all squares

C. $P$ is a full-infinite (AW) cone, and for every $p \in P, 1+p$ has an inverse in $P$.

D. A is a field, $P$ is a primitive (AW) cone containing $(1+p)^{-1}$ for every $p$ in $P$.

E. $A$ is a simple ring and $P$ is full Archimedean.

Corollary 2. Let $F$ be a field, let $P$ be a conic prime in $F$ whose intersection $P_{C}$ in the center $C$ of $F$ is a primitive (AC) cone in $C$. Then $P_{C}$ is an Archimedean order in $C$. 
Corollary 3. Let $F$ be a field with center $C$, let $P$ be an (AC) cone in $F$, and for each $x$ in $F$ let $P_{x}=P \cap C(x)$, where $C(x)$ is the extension field of $C$ by $x$. Suppose for each $x, P_{x}$ is a full-infinite prime in $C(x)$. Then $P$ is an Archimedean order in $F$.

THEOREM 2. If $P$ is an infinite $N$-cancellation (GAC) preprime in the ring $A$ then $P$ contains no large elements. If $P$ is a nonzero prime, if $P \cap(-P)=\{0\}$ and if $P$ contains no large elements, then $P$ is a (GAC) N-cancellation cone.

Theorem 3. Let $F$ be a field, let $P$ be a full Archimedean $N$ cancellation cone in $F$. Then $\langle F, P\rangle$ is a Stone ring, $P$ is a division cone, $F$ is isomorphic (algebraically) with a subfield of the reals, and the space $X_{F}$ of Theorem 09 is totally disconnected with a base of power not exceeding $c=2^{\aleph_{0}}$ of open-and-closed sets. Conversely, if $X$ is a totally disconnected compact Hausdorff space with a base of power at most $c$ of open-and-closed sets then $C(X)$ contains a dense subfield.

Corollary. Let $X$ be a compact Hausdorff space. Then $X$ admits a base of power at most $c$ of open-and-closed sets if and only if $C(X)$ has a dense subfield.

\section{b. Definitions.}

Let $P$ be an infinite preprime in the ring $A$ with unity 1 ; that is $P$ contains zero and one and is closed under addition and multiplication. $P$ is conic (or a cone) when $P \cap(-P)=\{0\}$. We denote by $N$ the set of all positive integers. We say $P$ is an $N$-cancellation preprime when from $n x$ in $P$, where $n \in N, x \in A$, follows $x \in P . P$ is full or full-infinite (or real-infinite) whenever $P-P=\left\{p_{1}-p_{2}\right.$; $\left.p_{1}, p_{2} \in P\right\}$ is equal to $A$. We define sets:

$$
\begin{aligned}
& P^{*}=\{x \in A ; \text { for all } n \in N, 1+n x \in P\}, \\
& B_{P}=\{x \in A ; \text { for some } n \in N, n \pm x \in P\}, \\
& J_{P}=\{x \in A ; \text { for all } n \in N, 1 \pm n x \in P\} .
\end{aligned}
$$

Elements of $B_{P}$ are called bounded elements, those of $J_{P}$ are infinitesimal. Observe that $P \cap(-P) \subset J_{P}=P^{*} \cap\left(-P^{*}\right) \subset B_{P}$. If $P$ is an $N$-cancellation preprime (more generally, if $2 x \in P$ implies $x \in P$ ) then $B_{P}$ is a subring and $J_{P}$ is an ideal in $B_{P}$; see [4; §2]. $P$ is (AC), or Archimedean in the sense of Clifford, when $P^{*}=P$, is $(\mathrm{AW})$, or Archimedean in the weak sense, when $J_{P}=\{0\}$. From the observation just above we see that an (AW) preprime is conic. Call P Archi- 
medean whenever $P \subset B_{P}$. In our first note we used "Archimedean" to mean what we are now calling "full Archimedean"; thus a Stone ring is a pair $(A, P)$ where $P$ is an infinite conic preprime which is full, Archimedean, and (AC). To say that $P$ is a full Archimedean preprime is equivalent to the condition $A=B_{P}$. An element $p$ (necessarily in $P$ ) is large if $p-n$ belongs to $P$ for all $n$ in $N . \quad P$ is (GAC), where $G$ stands for 'generalized', provided for each $p$ in $P$, the set $\{x$; for all $n$ in $N, p+n x \in P\}$ is contained in $P$ (therefore equal to $P$ ). In the special case that $A$ is a field (commutative or not) the preprime $P$ in $A$ is primitive if it is not contained in any proper subfield of $A$ (this is weaker than full, for if $P$ is full then it is contained in no proper subring of $A$ ), and $P$ is a division preprime if it contains the reciprocal of each of its nonzero members. In the general ring situation again, we call $P$ an order if it is conic and $P \cup(-P)=A$. Our Theorem 2.1 of [4] becomes: If $P$ is a division cone in the commutative field $A$ then $P$ is primitive if and only if $A$ is the field of quotients of $B_{P}$. For an order $P$ in a field $A$ all these Archimedeanlike conditions are equivalent to the condition that $P$ contains no large elements. If $P$ is full Archimedean in $A$ then we denote by $P / J$ the set of all cosets $y$ in $A / J\left(J=J_{P}\right)$ such that $y$ contains a member of $P$.

3. Proof of Theorem $\mathbf{1}$ and its corollaries. Let $P$ be an (infinite) $N$-cancellation preprime in a ring $A$. [For example, let $O$ be a non-Archimedean order in a field $F$, and let $p$ be a large element. Then $p$ and $-p^{-1}$ belong to $O^{*}$ but their product -1 does not. Thus $P^{*}$ is not always closed under multiplication.] We state without proof some easy properties of $P^{*}$ : (1) for each $n_{1}$ and $n_{2}$ in $N, P^{*}=\{x \in A$; for all $\left.n \in N, n_{1}+n\left(n_{2} x\right) \in P\right\}$; (2) -1 is not in $P^{*}$; (3) $P^{*}$ contains $P$ and is additively closed; (4) if $P^{*}$ is a preprime then $B_{P^{*}}=B_{P}$, $J_{P^{*}}=J_{P}$ and $P^{*}$ is an infinite $N$-cancellation (AC) preprime, which is conic if and only if $P$ is (AW). These are due to Harrison [8].

A. Next suppose that $P$ is Archimedean, still assuming that $P$ is an infinite $N$-cancellation preprime. We have to prove that $P^{*}$ is an Archimedean $N$-cancellation preprime. By (2) and (3), proof that $P^{*}$ is a preprime requires only closure under multiplication. Let $x$ and $y$ be members of $P^{*}$. By (2) and (3) again, $x+y$ belongs to $P^{*}$ and so $1+x+y$ is in $P$. By the Archimedean property of $P$ there exists $m$ in $N$ with $m-1-x-y$ in $P$. Let $n$ be arbitrary in $N$. Then $1+n x, 1+n y$ and $n-1$ all belong to $P$ and consequently $n(1+x+$ $y+n x y)=n-1+(1+n x)(1+n y)$ is also in $P$. From the left side of this equality cancel $n$ and then add $m-1-x-y$ to get $m+n x y$ in $P$. By (1) above, $x y$ belongs to $P$. If $x$ is any member of $P^{*}$ 
then $1+x$ is in $P$ and there exists $m$ in $N$ with $m-1-x$ in $P$. This implies that $m-x$ belongs to $P$, hence to $P^{*}$; hence $P^{*}$ is Archimedean. If $P$ is full Archimedean then $P^{*}$ is clearly also full Archimedean since $B_{P^{*}}=B_{P}=A$. The rest of part $A$ is included in (4) above, now that we have shown that $P^{*}$ is a preprime.

B. Let $P$ be a full Archimedean $N$-cancellation preprime in $A$. From what we just proved we may assume that $P$ is also (AC). Put $J=J_{P}, B=B_{P}$. Let $x$ be an element of $P$ and suppose $x-y$ belongs to $J$. Then for all $n$ in $N, 2+n y=1+n x+(1+n(y-x))$ belongs to $P$. By (1), and the (AC) condition on $P, y$ is also in $P$. Hence a coset belongs to $P / J$ if and only if all its members belong to $P$. It follows that -1 is not in $P / J$, while closure under addition and multiplication is obvious. Thus $P / J$ is an (infinite) preprime. If $n-x$ belongs to $P$, for $x$ in $A$ and $n$ in $N$ then $n-(x+J)$ belongs to $P / J$. Hence $P / J$ is full Archimedean. If $1+n(x+J)$ belongs to $P / J$ for all $n$ then $1+n x \in P$ for all $n$. Since $P$ is (AC) this implies $x$ in $P$ and so $x+J$ belongs to $P / J$; thus $P / J$ is (AC). The same argument shows that if $1 \pm n(x+J)$ belongs to $P / J$ for all $n$ then $1+n x$ belongs to $P$ for all $n, x$ is thus in $J, x+J=0$. So $P / J$ is (AW) and is therefore a cone. We have shown that $\langle A / J, P / J\rangle$ is a Stone ring, completing the proof of part $B$.

C. Since $P_{1}=P \cap B$ is full Archimedean in $B$ the assertion $C$ is an immediate consequence of part $A$.

D. This is deduced from part $B$ by observing that an Archimedean $P$ is full Archimedean in the ring $P-P$.

Proof of Corollary 1. A. Since for any $x, 1+x^{2}$ is invertible we may write $x=x\left(1+x^{2}\right)^{-1}\left(1+x^{2}\right)$; putting $a=x\left(1+x^{2}\right)^{-1}, \quad b=$ $\left(1+x^{2}\right)^{-1}$, we have $x=a b^{-1}$. Theorem $1 C$ guarantees that $B$ is commutative. Hence the proof will be complete if we show that each of $a$ and $b$ belongs to $B$. Since $P$ contains all squares, $b=\left(1+x^{2}\right)^{-1}=$ $\left(1+x^{2}\right)^{-2}+x^{2}\left(1+x^{2}\right)^{-2}$ is positive which implies positivity of $1-b=$ $x^{2}\left(1+x^{2}\right)^{-1}$. Thus $1 \pm b \in P, b \in B$. Also $4\left[1 \pm x\left(1+x^{2}\right)^{-1}\right]=$ $\left(1+x^{2}\right)^{-1}\left(4 x^{2} \pm 4 x+4\right)=\left(1+x^{2}\right)^{-1}\left[(2 x \pm 1)^{2}+3\right] \in P$. By cancellation we see that $1 \pm a \in P, a \in B$.

B. Immediate from part A.

C. As in part $\mathrm{A}$, the representation $p=\left[p(1+p)^{-1} \|(1+p)^{-1}\right]^{-1}$ has the form $a b^{-1}$, with $a$ and $b$ in $B$, provided $p$ belongs to $P$; in fact, $0 \leqq(1+p)^{-1} \leqq 1,0 \leqq p(1+p)^{-1} \leqq 1$ is valid. This shows that the elements of $P$ commute with each other. By the full-infinite property, any two elements $x$ and $y$ can be written $x=p-q, y=p^{\prime}-q^{\prime}$, $p, q, p^{\prime}, q^{\prime}$ all in $P$. But then certainly $x y=y x$. Hence $A$ is commutative. 
D. The argument of part $C$ shows that if $M$ is the smallest subring of $A$ containing $P$ then $M$ is commutative, and so, therefore, is its field of quotients. Since $P$ is primitive this last field is $A$.

E. The hypotheses imply that $A=B$. Since $J$ is an ideal in the simple ring $B$, and 1 is not in $J, J$ is null. That is, $P$ is (AW) so Theorem 1C applies to show that $A=B$ is commutative.

Proof of Corollary 2. For each $s$ in the center of $F$, let

$$
P\langle s\rangle=\left\{p+q s ; p, q, q s^{2} \text { belong to } P\right\} \text {. }
$$

Then simple computations show that $P\langle s\rangle$ is an additively and multiplicatively closed subset of $F$ containing $P$. We show that for every $s$ in $C$, at least one of $P\langle s\rangle$ and $P\langle-s\rangle$ is equal to $P$. Suppose both contain $P$ properly so that -1 belongs to both. From membership of -1 in $P\langle s\rangle$ follows $-1=p+q s$ for some $p, q$ in $P, q \neq 0$. Hence $s=-q^{-1}(1+p)=-(1+p) q^{-1}$. Since also -1 belongs to $P\langle-s\rangle$, we get $-s=-v^{-1}(1+u)=-(1+u) v^{-1}$ for some $u, v$ in $P, v \neq 0$. Combining these we find $(1+u) q=-v(1+p)$, so $(1+u) q$ belongs to $P \cap(-P)$, hence is zero. But $q$ is not zero so $u=-1$, contrary to $u \in P$.

If $p$ is any nonzero member of $P_{C}$ then $-p$ is outside $P$ so by Theorem 07, $p^{-1}$ belongs to $P$, and in fact $p^{-1}$ belongs to $P_{C}$. Thus $P_{C}$ is a division cone in $C$. Since $P_{C}$ is also a primitive (AC) cone Theorem 05 applies to guarantee that $P_{C}$ contains all squares in $C$, whence $P\langle s\rangle=\{p+q s ; p, q \in P\}$. For every $s$ in $C, s=0+1 s$ belongs to $P\langle s\rangle$. The italicized statement of the first paragraph shows that for every $s$, either $s$ or $-s$ belongs to $P_{C}(s$ in $C)$; this means that $P_{C}$ is an order in $C$. The (AC) condition implies that $P_{C}$ is an Archimedean order in $C$.

Proof of Corollary 3. Let $x \in F$. The hypotheses imply that $P_{x}$ is a full-infinite $(\mathrm{AC})$ conic prime in $C(x)$. By Corollary $2, P_{x}$ is an Archimedean order in $C(x)(C(x)$ is commutative). If $x$ is any member of $F$, there accordingly exists an integer $n$ with $n-x$ in $P_{x} \subset P$. This implies that $P$ is itself Archimedean. Moreover, either $x$ or $-x$ belongs to $P_{x}$, and $P$ is therefore an order.

4. Proof of Theorem 2. The first claim is trivial, since if $p>n$ holds for all $n$ in $N$ then $p+n(-1) \in P$ for all $n$ so, by (GAC), $-1 \in P$, contradiction. Now let $P$ be a prime with $P \cap(-P)=(0)$, $P \neq\{0\}$. Then $P$ is not finite, since finite primes are additive subgroups according to Theorem 06 . Hence 1 belongs to $P$, and $n \cdot 1$ for $n \in N$, cannot be zero, since $n \cdot 1=0$ implies $-1=(n-1) \cdot 1 \in P$. Let $P^{\prime}$ be the set of all $x$ in $A$ such that for some $n$ in $N, n x \in P$. This 
is obviously closed under addition and multiplication. From -1 in $P^{\prime}$ follows $n(-1) \in P$ for some $n$. But $n \cdot 1 \in P$ so $n \cdot 1=0$, contrary to what was just proved. So $P^{\prime}$ is a preprime which contains the prime $P$. Thus $P^{\prime}=P$ and we have proved that $P$ is an $N$-cancellation cone. That is, if $P$ is a nonzero prime and $P \cap(-P)=(0)$, then $P$ is infinite and is an $N$-cancellation cone. Now suppose further that there are no large elements in $P$. In order to deduce (GAC) it is necessary and sufficient to show that the set $P_{1}$ of all $x$ such that for some $p$ in $P, p+n x \in P$ holds for all $n$ in $N$, is equal to $P$. If $x$ belongs to $P$ then $0+n x$ belongs also to $P$ for all $n$ so $P$ is contained in $P_{1}$. Next we show that $P_{1}$ is a preprime and the proof is then complete, since $P$ is a prime. Let $x$ and $y$ be members $P_{1}$, say $p+n x$, $q+n y$ belong to $P$ for all $n$ and $p$ and $q$ belong to $P$. For all $n$, $p+q+n(x+y)$ belongs to $P, p+q$ belongs to $P$, so $x+y$ is in $P_{1}$. Also members of $P$ are $p q+n x q=(p+n x) q, p q+n p y=p(q+n y)$ and $p q+n(x q+p y)+n^{2} x y=(p+n x)(q+n y)$, for all $n$ in $N$; putting $n=2$ in the first two expressions we see that $2(p q+x q+p y)=$ $(p q+2 x q)+(p q+2 p y)$ belongs to $P$. By cancellation, $p q+x q+p y$ is in $P$. Also $n p q+n(x q+p y)+n^{2} x y \geqq p q+n(x q+p y)+n^{2} x y$, the last belonging to $P$. Cancelling $n$ we get $p q+x q+p y+n x y$ in $P$. We just showed that $P$ contains $p q+x q+p y$, and $n$ is arbitrary. Hence $x y$ belongs to $P_{1}$. Finally, if -1 belonged to $P_{1}$ then for some $p$ in $P$ we would have $p+n(-1) \in P$ for all $n$, which would imply $p>n$ for all $n$, and this asserts that $p$ is large, contrary to our hypothesis that $P$ contains no large elements. This completes the proof that $P_{1}$ is a preprime and the theorem is proved.

5. Proof of Theorem 3. Since $F$ is a field, the ideal $J$ in $F=$ $B_{P}$ is null, and $\left\langle F, P^{*}\right\rangle$ is a Stone ring by Theorem 1B. Identify $F$ with a subfield of $C(X), X=X_{F}$. Then $F$ is dense in $C(X), P$ is dense in $P^{*}$. Since $F$ is a field, every nonzero member of $F$ vanishes nowhere in $X$. If $f$ is a nonzero member of $P^{*}$ then, by compactness of $X$, there exists $m$ in $N$ such that $f(x)>1 / m$ holds for all $x$ in $X$. Since $P^{*}$ is the set of all nonnegative functions in $F, f-1 / m$ belongs to $P^{*}$ and hence $f=(f-1 / m)+1 / m$ belongs to $P$. This shows that $P^{*}=P$. Clearly $P^{*}$ is a division cone in $F$. Let $x_{0}$ be fixed in $X$ and define $\varphi, F \rightarrow R=$ reals, by $\varphi(f)=f\left(x_{0}\right)$. This is a field monomorphism which establishes an isomorphism of $F$ with the subfield $\varphi(F)$ of $R$. Let $x_{1}$ and $x_{2}$ be distinct points of $X$. The subring $F$, as a dense subring of $C(X)$ containing the rational constant functions, separates points in $X$, since $X$ is a compact Hausdorff space. Hence there exists a function $f$ in $F$ with $f\left(x_{1}\right) \neq f\left(x_{2}\right)$, and so there is a rational $r$ between $f\left(x_{1}\right)$ and $f\left(x_{2}\right)$. But $f-r$ is a continuous function on $X$ 
which has opposite signs at $x_{1}$ and $x_{2}$, but has no zeros. Thus no connected subset of $X$ contains both $x_{1}$ and $x_{2}$, and we have proved that $X$ is totally disconnected. By compactness, $X$ has a base of open-and-closed sets [6; Th. 16.17]. Let $T$ be such a base. For every open set $G$ in $T$, the characteristic function of $G$ is continuous so we see that the power of $C(X)$ is at least as great as the power of $T$. But $C(X)$, as completion of the metric space $F$, has power at most $c^{\aleph_{0}}=c$, since $|F| \leqq c(F$ is a subfield of $R$ ). We have shown that every base of open-and-closed sets has power at most $c$.

Now for the converse, let $T$ be a base of power at most $c$ of openand-closed sets in a compact Hausdorff space $X$. Let the members of $T$ be indexed by ordinals:

$$
G_{1}, G_{2}, \cdots, G_{\xi}, \cdots ;(\xi<\Lambda)
$$

where $\Lambda$ is the least ordinal with the same power as $T$, and let

$$
t_{11}, t_{12}, t_{21}, t_{22}, \cdots, t_{\xi 1}, t_{\xi 2}, \cdots ;(\xi<\Lambda)
$$

be a well-ordered algebraically independent sequence of transcendental reals. For each $\xi$ define $f_{\xi}$ by the formula: $f_{\xi}(x)=t_{\xi 1}$ if $x$ belongs to $G_{\xi}, f_{\xi}(x)=t_{\xi 2}$ if $x$ is not in $G_{\xi}$. These functions are continuous. Let $D$ be the subring of $C(X)$ generated by all the $f_{\xi}$ and the rationalvalued constant functions. To show that $D$ is dense in $C(X)$ we need only show that $D$ separates points in $X$ and apply one form of the Stone-Weierstrass theorem. If $x_{1} \neq x_{2}$ then for some $\xi, x_{1}$ belongs to $G_{\xi}$ and $x_{2}$ does not. Then $f_{\xi}\left(x_{1}\right)=t_{\xi 1}, f_{\xi}\left(x_{2}\right)=t_{\xi 2} \neq t_{\xi 1}$. Thus $D$ separates points. It remains only to show that no nonzero member of $D$ has any zeros and then put $F$ equal to the field of quotients of $D$ in $C(X)$. Let $f$ be a member of $D$. Then $f$ is a polynomial in the $f_{\xi}$ with rational coefficients. An equality $f(x)=0$, for $x$ in $X$, implies an algebraic relation among the $f_{\xi}(x)$. But for any $x$, the $f_{\xi}(x)$ are algebraically independent, as distinct entries in the well ordered list $t_{11}, t_{12}, \cdots$. Hence if $f(x)=0$ then all the rational coefficients for $f$ are zero, $f=0$.

Proof of the corollary. Obvious in view of Theorem 3 and the uniqueness assertion of Theorem 09.

6. Examples. We recall briefly Hilbert's construction $19 ; \S 33]$. Let $F$ be a field (not necessarily commutative), let $P$ be an infinite preprime in $F$, let $\sigma$ be an automorphism of $F$ such that $\sigma(x)$ belongs to $P$ if and only if $x$ belongs to $P$. In the additive group of all formal Laurent series $\sum a_{k} t^{k}$ with only finitely many negative powers, the usual multiplication is altered as follows: instead of assuming that 
the indeterminate $t$ commutes with every element of $F$ we make $t^{k} a=\sigma^{k}(a) t^{k}$ for all integers $k$, and all $a$ in $F$. The resulting structure is a field $H=H(F, \sigma)$ which is noncommutative unless $F$ is commutative and $\sigma$ is the identity. In $H$ set $P_{1}$ equal to the set of all series whose lowest coefficient belongs to $P$ and denote by $P_{2}$ the set of all series whose lowest coefficient belongs to $P$. Then always $P_{2}$ is properly contained in $P_{1}$, and both are infinite preprimes. If $P$ is conic then so are $P_{1}$ and $P_{2}$; if $P$ is an order then $P_{1}$ is an order but $P_{2}$ is never an order (nor even a prime). Suppose now that $P$ is a cone. Then neither $P_{1}$ nor $P_{2}$ can be Archimedean while $P_{1}$ can never be $(\mathrm{AC}): 1+n(-t)$ belongs to $P_{1}$ for all $n$ but $-t$ is not in $P_{1}$. In case $P$ is an order and $\sigma$ is not the identity, then $P_{1}$ gives a (non-Archimedean) ordering of the noncommutative field $H$. This is Hilbert's example. Note further that if $P$ is full then so are $P_{1}$ and $P_{2}$. In the examples below $Q$ is the field of all rational numbers.

Example 1. Take $F=Q(\sqrt{2})$, let $P$ be the intersection of the two distinct orders in $F: P=\{a+b \sqrt{2} ; a \pm b \sqrt{2}$ are positive reals $\}$, and let $\sigma(a+b \sqrt{2})=a-b \sqrt{2}$. Then $H$ is noncommutative and $P_{2}$ is a full-infinite (AC) $N$-cancellation cone in $H$.

Example 2. The set $P$ of all $n^{\times} n$ matrices with every entry a nonnegative real is a full-infinite (AC) conic $N$-cancellation prime in the noncommutative simple ring $A$ of all real $n^{\times} n$ matrices. It is not Archimedean.

Example 3. The set $P$ of all positive reals in the field $U_{R}$ of all real quaternions is an Archimedean $N$-cancellation (AC) conic prime in a noncommutative field. It is not full-infinite.

EXAmple 4 . The set $P$ of all lower triangular $n^{\times} n$ matrices containing the zero matrix and all those with strictly positive real entries on the diagonal is an Archimedean full-infinite $\mathrm{N}$-cancellation cone in the noncommutative ring of all real lower triangular $n^{\times} n$ matrices. $P$ is not (AW) since every nilpotent element is infinitesimal.

Example 5. (Dubois [4].) Let $F$ be a commutative field, let $P$ be an $N$-cancellation cone in $F$ with $J_{P} \neq\{0\}$, and let $\theta$ be integral over $B_{p}$ of degree $n$. In $F(\theta)$,

$$
P(\theta)=\left\{p\left(1+\sum_{i=1}^{n-1} a_{i} \theta^{i}\right) ; p \in P, a_{i} \in J_{P}\right\}
$$

is an $\mathrm{N}$-cancellation cone. If $P$ is full-infinite or a division cone then 
so is $P(\theta)$. For the special case of the following example, more is true:

EXAmPle 6. (Harrison [7].) Let $P$ be a non-Archimedean order in the field $F$, let $\theta=i, i^{2}=-1$. Then $P(i)$ is a prime in $F(i)$. Example 8 shows that $P$ can be a prime while $P(\theta)$ is not a prime. But the following is plausible: with the notation of Example 5, let $P$ be an order and suppose $F(\theta)$ is not formally real (i.e., suppose $F(\theta)$ cannot be ordered). Then $P(\theta)$ is (we conjecture) a prime.

Example 7. With $F$ and $P$ as in Example 6 , let $U_{F}$ be the field of quaternions over $F$, with generators $1, k_{1}, k_{2}, k_{3}, k_{i}^{2}=-1$, and let

$$
P^{\prime}=\left\{p\left(1+\sum a_{i} k_{i}\right) ; a_{i} \in J_{P}\right\} .
$$

Then $P^{\prime}$ is a full-infinite $N$-cancellation conic division prime in $U_{F}$.

Note. By Theorem 07, for a prime in a field the division and conic properties are equivalent.

ExAmPLE 8. Let $F=Q(x)$, where $x$ is an indeterminate and let $P$ be the order containing $x$ as an infinitesimal. The cone $P(\sqrt{2})$ of of Example 5 is not a prime since it is properly contained in the order $P_{0}$ in $Q(x, \sqrt{2})$ which induces the usual (real) ordering of $Q(\sqrt{2})$ and contains $x$ as an infinitesimal.

An immediate corollary of Theorem 05 is that any primitive (AC) conic division prime in a commutative field is an Archimedean order. Corollary 2 generalizes this as follows: Let $P$ be a primitive (AC) conic prime in a commutative field $F$. Then $P$ is an Archimedean order in $F$.

EXAMPLE 9. Let $F$ be the (commutative) field of all formal Laurent series in $x$, with coefficients in $Q$ and let $P$ be the set of all such series with all coefficients nonnegative. Then $P$ is a primitive (in fact a full-infinite)(AC) cone in $F$. But $\left(1+x^{2}\right)^{-1}$ is a sum of squares that is not in $P$. Thus the division condition cannot be deleted from Theorem 05 .

ExAMPle 10. Let $F$ and $P$ be the same as in Example 9, let $Q(x)$ be the subfield of all rational functions in $F$, and let $S=$ $P \cap Q(x)$. Then $S$ is a full-infinite $(\mathrm{AC})$ cone in $Q(x)$. But $Q(x)$ is not the field of quotients of $B_{S}$; in fact $B_{S}=Q$. 


\section{REFERENCES}

1. E. Artin, Über die Zerlegung definiter Funktionen in Quadrate, Abh. Math. Sem. Univ. Hamburg 5 (1927), 100-115.

2. E. Artin and O. Schreier, Algebraische Konstruktion reeler Körper, Abh. Math. Sem. Univ. Hamburg 5 (1927), 85-99.

3. R. Baer, Über nichtarchimedisch geordnete Körper, S.-B. Heidelberger Akad. Wiss. Math. Natur. Kl. 8 (1927).

4. D. W. Dubois, On partly ordered fields, Proc. Amer. Math. Soc. 7 (1956), 918-930. 5. _ A note on David Harrison's theory of preprimes, Pacific J. Math. 21 (1967), 15-19.

6. L. Gillman and M. Jerison, Rings of continuous functions, Princeton, 1960.

7. D. K. Harrison, Finite and infinite primes for rings and fields, mimeographed preprint.

8. S Seminar on rings and fields, unpublished notes of a seminar given at the University of Oregon, 1965-1966.

9. D. Hilbert, Grundlagen der Geometrie, Stuttgart, 1956.

10. R. V. Kadison, A representation theory for commutative topological algebra, Mem. Amer. Math. Soc. 7 (1951).

11. M. H. Stone, A general theory of spectra, I, Proc. Nat. Acad. Sci. U.S.A. 26 (1940), $280-283$

Received July 22, 1966.

University of NeW Mexico 


\section{PACIFIC JOURNAL OF MATHEMATICS}

\section{EDITORS}

\section{H. ROYDEN}

Stanford University

Stanford, California

\section{J. P. Jans}

University of Washington

Seattle, Washington 98105

\section{J. DUGUNDJI}

Department of Mathematics

Rice University

Houston, Texas 77001

RICHARD ARENS

University of California

Los Angeles, California 90024

\section{ASSOCIATE EDITORS}
E. F. BeCKenbaCH
B. H. NeumanN
F. WOLF
K. YosIDA

\section{SUPPORTING INSTITUTIONS}

UNIVERSITY OF BRITISH COLUMBIA CALIFORNIA INSTITUTE OF TECHNOLOGY UNIVERSITY OF CALIFORNIA MONTANA STATE UNIVERSITY UNIVERSITY OF NEVADA NEW MEXICO STATE UNIVERSITY OREGON STATE UNIVERSITY UNIVERSITY OF OREGON OSAKA UNIVERSITY UNIVERSITY OF SOUTHERN CALIFORNIA
STANFORD UNIVERSITY UNIVERSITY OF TOKYO UNIVERSITY OF UTAH WASHINGTON STATE UNIVERSITY UNIVERSITY OF WASHINGTON

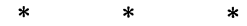

AMERICAN MATHEMATICAL SOCIETY CHEVRON RESEARCH CORPORATION TRW SYSTEMS

NAVAL ORDNANCE TEST STATION 


\section{Pacific Journal of Mathematics}

\section{Vol. 24, No. $1 \quad$ May, 1968}

Harry P. Allen, Lie algebras of type $D_{4}$ over algebraic number fields ...... 1

Charles Ballantine, Products of positive definite matrices. II............ 7

David W. Boyd, The spectral radius of averaging operators ............ 19

William Howard Caldwell, Hypercyclic rings ................... 29

Francis William Carroll, Some properties of sequences, with an application

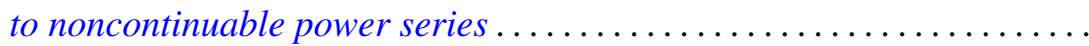

David Fleming Dawson, Matrix summability over certain classes of

sequences ordered with respect to rate of convergence ........... 51

D. W. Dubois, Second note on David Harrison's theory of preprimes. . . . . 57

Edgar Earle Enochs, A note on quasi-Frobenius rings.............. 69

Ronald J. Ensey, Isomorphism invariants for Abelian groups modulo bounded groups ................................ 71

Ronald Owen Fulp, Generalized semigroup kernels ................ 93

Bernard Robert Kripke and Richard Bruce Holmes, Interposition and approximation ................................. 103

Jack W. Macki and James Sai-Wing Wong, Oscillation of solutions to second-order nonlinear differential equations ..................

Lothrop Mittenthal, Operator valued analytic functions and generalizations

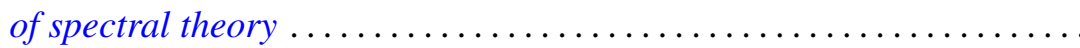

T. S. Motzkin and J. L. Walsh, A persistent local maximum of the pth power deviation on an interval, $p<1 \ldots \ldots \ldots \ldots \ldots \ldots \ldots \ldots \ldots \ldots . \ldots \ldots$

Jerome L. Paul, Sequences of homeomorphisms which converge to homeomorphisms ...........................

Maxwell Alexander Rosenlicht, Liouville's theorem on functions with elementary integrals.

Joseph Goeffrey Rosenstein, Initial segments of degrees .

$\mathrm{H}$. Subramanian, Ideal neighbourhoods in a ring ............

Dalton Tarwater, Galois cohomology of abelian groups . .

James Patrick Williams, Schwarz norms for operators ... .

Raymond Y. T. Wong, A wild Cantor set in the Hilbert cube. 